\title{
Associations of Self-Consciousness with Insomnia Symptoms
}

\author{
Min Su Kim ${ }^{1,2}$, Eun Lee ${ }^{1,2}$, Hye Yoon Park ${ }^{1,2}$, Eunchong Seo ${ }^{1,2}$, Suk Kyoon An ${ }^{1,2}$, and Kyung Mee Park ${ }^{1,2,3}$ \\ 'Department of Psychiatry, Severance Hospital, Yonsei University College of Medicine, Seoul, Korea \\ 2Institute of Behavioral Science in Medicine, Severance Hospital, Yonsei University College of Medicine, Seoul, Korea \\ ${ }^{3}$ Department of Hospital Medicine, Yongin Severance Hospital, Yonsei University College of Medicine, Yongin, Korea
}

\begin{abstract}
Objective: Insomnia is a very common health problem that are getting more attention. Self-consciousness is one of the factors related to anxiety or rumination that affect insomnia symptoms. However, the number of studies regarding self-consciousness and insomnia is scarce. The objective of study is to evaluate effects of self-consciousness on insomnia symptoms. Methods: A total of 90 healthy adults (mean age 28 years, 58\% female) were enrolled to the study. Pittsburgh Sleep Quality Index (PSQI) and Insomnia Severity Index (ISI) were used to define poor sleepers (PSQI score 8 or higher, or ISI score 8 or higher). Objective sleep parameters were obtained through actigraphy device, and subjective sleep parameters were obtained through sleep diaries concordantly. Self-consciousness scale was used to evaluate three domains of selfconsciousness including public self-consciousness, private self-consciousness, and social anxiety. Binary regression analysis and multiple logistic regression analysis were conducted to find correlation between self-consciousness and insomnia symptoms. Results: Among three selfconsciousness domains, only social anxiety showed significant correlation to poor sleepers [odds ratio $(O R)=1.091, p=0.046]$. Social anxiety was also significantly correlated with both PSQI score 8 or higher $(B=0.091, p=0.002)$ and ISI score 8 or higher $(B=0.087, p=0.047)$. Private self-consciousness was negatively associated with ISI score 8 or higher $(B=-0.202, p=0.043)$. Conclusion: High social anxiety had a significant correlation to poor sleep. The result of this study implies high self-consciousness with emotional distress could affect insomnia symptoms.

Key Words: Insomnia; Sleep; Self-consciousness; Social anxiety; Actigraphy; Sleep diary

Received: March 6, 2021 Revised: March 9, 2021 Accepted: March 10, 2021

Corresponding author: Kyung Mee Park, MD, PhD, Department of Hospital Medicine, Yongin Severance Hospital, Yonsei University College of Medicine, 363 Dongbaekjukjeon-daero, Giheung-gu, Yongin 16995, Korea.

Tel: 82-31-5189-8531, Fax: 82-31-5189-8565, E-mail: kmpark87@yuhs.ac

(a) This is an Open Access article distributed under the terms of the Creative Commons Attribution Non-Commercial License (https://creativecommons.org/licenses/bync/4.0) which permits unrestricted non-commercial use, distribution, and reproduction in any medium, provided the original work is properly cited.
\end{abstract}

\section{INTRODUCTION}

Insomnia is a common health problem that have recently been getting more attention. According to a 2017 Korean Gallup survey on 1004 Korean adults, 34 percent of people reported that they did not sleep well recently [1], and World Health Organization Study on Global Ageing and Adult Health claimed that 150 million of adults from 8 countries have sleep problem in 2007, and the numbers are expected to be increased more than 260 million in 2030 [2]. Insomnia symptoms including initiating or maintaining sleep, short sleep duration, and poor sleep quality are known to have correlation with cardiovascular disease risk, overall mortality, poor psychiatric condition, or daytime functioning including cognitive performance [3-8].

Self-consciousness, which is defined as one of the adaptive per- sonality processes that are the object of one's consciousness which accompanies the natural disposition of, is one of the factors related to anxiety or rumination that affect insomnia symptoms [911]. Self-consciousness is generally considered to have two dimensions; public and private aspects of self-consciousness [12]. Public self-consciousness is outward directions of one's thought, while private self-consciousness is inward directions of one's thought. Social anxiety, discomfort in the presence of others, is a reaction to self-focused attention which also have theoretical implications for the nature of self-consciousness [12]. In the aspects of self-consciousness, social anxiety is considered as an enfoldment of public self-consciousness [13]. Self-consciousness is related to insomnia because people with insomnia are generally have self-consciousness about their sleep [10], yet the number of studies regarding self-consciousness and insomnia are scarce. 
Some researchers reported the association between sleep problems and counterfactual emotions that included self-consciousness, such as social anxiety, regret, guilt, and shame [14-16]. However, there is still a lack of research on the effects of self-consciousness on insomnia.

In this study, we tried to find correlation between self-consciousness and insomnia symptoms. We hypothesized that selfconsciousness would have negative effect on insomnia symptoms. We measured objective and subjective sleep parameters of the participants, and performed analysis to find the effects of self-consciousness on insomnia.

\section{METHODS}

\section{Study population}

Target population was healthy adults aged more than 19 years. We recruited participants through advertisements posted at Severance Hospital and Yonsei University College of Medicine. Exclusion criteria were as below; current or previous major psychiatric disorder (e.g., schizophrenia or related psychotic disorder, bipolar disorder, major depressive disorder, and anxiety disorder), substance use disorder (except for nicotine), uncontrolled medical condition, and regular use of sleeping medication. All of the participants had given explanation of the research purposes and procedure beforehand, and provided written consent voluntarily. The study was approved by the Institutional Review Board of Severance Hospital, Yonsei University Health System (no: 42017-0893 and 4-2016-0570).

\section{Baseline measurement}

Baseline characteristics including sex, age, job status (regular worker, part-time job, housemaker, student, or inoccupation), education level (years), presence of current psychiatric disorder, and other medical condition were obtained through face-to-face interview. Presence of psychiatric disorder was evaluated using the Structured clinical interview for DSM-5 (SCID-5) [17].

For measuring baseline sleep status, Pittsburgh Sleep Quality Index (PSQI) and Insomnia Severity Index (ISI) were used. PSQI is a self-rated questionnaire that assesses sleep quality and disturbances during past month [18]. It consists of 7 components including subjective sleep quality, sleep latency, sleep duration, habitual sleep efficiency, sleep disturbances, use of sleeping medication, and daytime dysfunction based on experience within 1 month of patients' experience. Each component scored from 0 point to 3 point, and the sum of scores for these seven components yields one global score for PSQI. Higher PSQI score implies worse sleep status. Cut-off score of PSQI is generally considered as 5, which implies poor sleep quality $[18,19]$, while others suggest a cut-off score of 8 is more suitable depending on the sample characteristics [20,21]. ISI is a 7-item self-report questionnaire evaluating below items; severity of sleep-onset, sleep maintenance, early morning awakening problems, satisfaction with current sleep pattern, interference with daily functioning, noticeability of im- pairment attributed to the sleep problem, and level of distress caused by sleep problem [22]. Each of these items is rated on a five-point Likert scale (' 0 ' not at all, ' 4 '=extremely) based on experience within two weeks. High scores indicating greater insomnia severity. Cut-off score of ISI is 8, which suggesting sub-threshold insomnia [23]. In this study, if the participants' PSQI score was 8 or higher regardless of ISI score, or ISI score was 8 or higher regardless of PSQI score, all were defined as poor sleepers. If participants have both PSQI score lower than 8 and ISI score lower than 8 , they were defined as good sleepers.

\section{Study design}

After their initial meeting with researchers, participants were asked to continuously wear the actigraphy device (ActiGraph GT3X $+{ }^{\circledR}$, ActiGraph, Pensacola, FL, USA) on their non-dominant wrist for 14 days, and to write sleep diary during same periods. If they did not wear actigraphy or write sleep diary for more than 7 days, they were excluded from the study. After the experiment, the participants were asked to conduct a survey on selfconsciousness through e-mail.

Actigraphy was used to gather information of objective sleep parameters including objective sleep onset latency (SOL), objective total sleep time (TST), objective time in bed (TIB), and objective wake after sleep onset (WASO). Objective sleep efficiency was defined as ratio of objective TST per objective TIB. Actigraphy detects participants' movement as activity counts in every 60 second (1 epoch). All epochs were scored as either sleep or wake status by comparing the calculated total activity counts to awake threshold value based on Cole-Kripke algorithm [24]. If the total activity counts were less than or equal to the wake threshold value, the epoch was scored as sleep. ActiLife software (version 6.13.3, ActiGraph) was used to assess and analyze data from actigraphy.

International consensus format of sleep diary was used to measure subjective sleep parameters to assess subjective SOL, subjective TST, subjective TIB, subjective WASO, subjective sleep quality, consumption of alcohol, and number and time of naps [25]. Subjective sleep efficiency was defined as ratio of subjective TST per subjective TIB. We encouraged participants to complete the diary immediately upon awakening each morning to minimize errors.

Self-consciousness scale (SCS) was used to evaluate self-consciousness of participants [26]. It is self-rated questionnaire consisted with 23 questions assessing three domains of self-consciousness; private self-consciousness (nine items, e.g. "'I'm always trying to figure myself out."), public self-consciousness (seven items, e.g., "I'm concerned about the way I present myself."), and social anxiety (six items, e.g., "I have trouble working when someone is watching me.") [13]. The private self-consciousness means one's concern with attending to their inner thoughts and feelings. The public self-consciousness is defined by a general awareness of the self as a social object that has an effect on others. The third domain, social anxiety, is defined by a discomfort in the presence of others. Higher score indicates higher self-consciousness. 
Statistical analyses

A total of 106 individuals were enrolled to the study. Data from 16 participants were excluded from analysis for the following reasons; consent withdrawal $(\mathrm{n}=1)$, without use of actigraphy for more than 7 days $(n=1)$, without use of sleep diary for more than 7 days $(n=2)$, incompletion of self-questionnaire $(n=12)$. Therefore, data from a total of 90 people were used for the analysis.

To evaluate the effects of self-consciousness on insomnia symptoms, we performed independent sample t-test, binary regression analysis, and multiple logistic regression analysis. Independent sample t-tests were used to compare differences between poor sleepers (PSQI $\geq 8$ or $\mathrm{ISI} \geq 8$ ) and good sleepers (PSQI $<8$ and ISI $<8$ ) including demographic factors, subjective and objective sleep parameters, and self-consciousness. Binary regression analysis was conducted to evaluate the effects of factors, including self-consciousness, on poor sleepers. Multiple logistic regression analysis was used to evaluate the effects of factors on each insomnia scale. The presence of multicollinearity among variables in regression model was examined by variance inflation factor, and no factors were found to have multicollinearity problem. Statistical significance was set at $p<0.05$. All analyses were performed by using statistical package for social science 23 for windows version (IBM Corp., Armonk, NY, USA) [27].

\section{RESULTS}

\section{Participants characteristics}

A total of 90 participant including poor sleepers $(n=30)$ and good sleepers $(n=60)$ were included in the study. Demographic and clinical characteristics of the 90 study participants (aged 20 to 61 years) are shown in Table 1 . Subjective sleep quality was worse in poor sleepers than in good sleepers $(\mathrm{p}<0.001)$. There was a tendency of less subjective TST in poor sleepers than in good sleepers $(\mathrm{p}=0.050)$, whereas other sleep parameters including objective sleep parameters measured by actigraphy were not significantly different between two groups. Among the three domains of selfconsciousness, only social anxiety was significantly higher in poor sleepers than in good sleepers $(\mathrm{p}=0.010)$.

Table 1. Characteristics of study participants

\begin{tabular}{|c|c|c|c|c|}
\hline Characteristic & Total $(\mathrm{n}=90)$ & Poor sleepers ${ }^{\mathrm{a}}(\mathrm{n}=30)$ & Good sleepers $^{\mathrm{b}}(\mathrm{n}=60)$ & $\mathrm{p}$ \\
\hline Age (yr) & $28.73 \pm 8.53$ & $29.30 \pm 6.73$ & $28.45 \pm 9.336$ & 0.658 \\
\hline Sex, female & $53(58.9)$ & $21(70)$ & $32(53.3)$ & 0.130 \\
\hline Weight $(\mathrm{kg})$ & $63.04 \pm 12.52$ & $63.89 \pm 14.50$ & $62.61 \pm 11.491$ & 0.653 \\
\hline Education (yr) & $16.43 \pm 2.26$ & $17.03 \pm 1.99$ & $16.14 \pm 2.345$ & 0.080 \\
\hline Current job status & 84 & $28(33.3)$ & $56(66.7)$ & 0.109 \\
\hline Regular worker & $33(39.3)$ & $14(50)$ & $19(33.93)$ & \\
\hline Part-time job & $1(1.2)$ & $1(3.57)$ & 0 & \\
\hline Housemaker & $2(2.4)$ & $1(3.57)$ & $1(1.79)$ & \\
\hline Student & $47(56.0)$ & $11(39.29)$ & $36(64.29)$ & \\
\hline Inoccupation & $1(1.2)$ & $1(3.57)$ & 0 & \\
\hline Total nap time (min) & $21.44 \pm 20.42$ & $20.87 \pm 18.36$ & $21.73 \pm 21.510$ & 0.850 \\
\hline Amount of drinks (g) & $3.33 \pm 5.65$ & $3.36 \pm 5.55$ & $3.31 \pm 5.75$ & 0.966 \\
\hline Subjective SOL (min) & $13.06 \pm 11.05$ & $12.84 \pm 11.475$ & $13.17 \pm 10.94$ & 0.894 \\
\hline Subjective TST (min) & $407.38 \pm 56.30$ & $390.99 \pm 49.08$ & $415.58 \pm 58.25$ & 0.050 \\
\hline Subjective TIB (min) & $467.78 \pm 76.20$ & $448.22 \pm 72.99$ & $477.56 \pm 76.47$ & 0.085 \\
\hline Subjective WASO (min) & $4.58 \pm 4.69$ & $6.04 \pm 5.68$ & $3.84 \pm 3.97$ & 0.064 \\
\hline Subjective SE (\%) & $87.61 \pm 6.58$ & $87.81 \pm 6.26$ & $87.51 \pm 6.78$ & 0.839 \\
\hline Subjective sleep quality ${ }^{c}$ & $6.74 \pm 1.56$ & $5.98 \pm 1.47$ & $7.13 \pm 1.48$ & $<0.001^{\dagger}$ \\
\hline Objective SOL (min) & $1.27 \pm 0.76$ & $1.21 \pm 0.78$ & $1.30 \pm 0.76$ & 0.586 \\
\hline Objective TST (min) & $386.39 \pm 60.37$ & $378.12 \pm 48.49$ & $390.53 \pm 65.49$ & 0.314 \\
\hline Objective TIB (min) & $446.60 \pm 61.77$ & $437.20 \pm 54.60$ & $451.30 \pm 64.98$ & 0.310 \\
\hline Objective WASO (min) & $58.93 \pm 18.76$ & $57.87 \pm 21.06$ & $59.46 \pm 17.66$ & 0.707 \\
\hline Objective SE (\%) & $86.35 \pm 4.44$ & $86.53 \pm 4.50$ & $86.27 \pm 4.45$ & 0.790 \\
\hline PSQI (score) & $6.07 \pm 2.55$ & $8.77 \pm 1.78$ & $4.72 \pm 1.65$ & $<0.001^{\dagger}$ \\
\hline ISI (score) & $5.12 \pm 3.57$ & $8.83 \pm 3.19$ & $3.27 \pm 1.93$ & $<0.001^{\dagger}$ \\
\hline Private self-consciousness ${ }^{\mathrm{d}}$ & $32.58 \pm 5.24$ & $32.77 \pm 5.17$ & $32.48 \pm 5.32$ & 0.811 \\
\hline Public self-consciousness ${ }^{\mathrm{d}}$ & $22.22 \pm 5.09$ & $22.87 \pm 4.49$ & $21.90 \pm 5.38$ & 0.399 \\
\hline Social anxiety ${ }^{\mathrm{d}}$ & $38.01 \pm 15.17$ & $43.23 \pm 11.440$ & $35.40 \pm 16.196$ & $0.010^{*}$ \\
\hline
\end{tabular}

Values are expressed as mean \pm SD for continuous variables and as $\mathrm{n}(\%)$ for categorical variables. Subjective sleep parameters are measured by using sleep diary. Objective sleep parameters are measured by using actigraphy. ${ }^{*} \mathrm{p}<0.05,{ }^{\dagger} \mathrm{p}<0.01$. ${ }^{\mathrm{a}}$ Participants with PSQI $\geq 8$ or ISI $\geq 8$. ${ }^{\mathrm{b}} \mathrm{Participants}$ with PSQI $<8$ and ISI $<8$. ${ }^{~}$ Measured on a Likert scale, range from 1 to $10 .{ }^{d}$ Measured by Self-consciousness scale. SOL: sleep onset latency, TST: total sleep time, TIB: time in bed, WASO: wake after sleep onset, SE: sleep efficiency, PSQI, Pittsburgh Sleep Quality Index, ISI: Insomnia Severity Index 
Association between self-consciousness and poor sleep

Higher social anxiety was significantly associated with poor sleepers in binary regression analysis [odds ratio $(\mathrm{OR})=1.091, \mathrm{p}=$ 0.046] (Table 2). As shown in Table 3, we found that only social anxiety had significant effect on the poor sleepers with PSQI score of 8 or higher in multiple regression analysis $(B=0.091$, $\mathrm{p}=0.002$ ). On the other hand, both private self-consciousness and social anxiety showed significant effects on the poor sleepers with the ISI score of 8 or higher $(\mathrm{B}=-0.202, \mathrm{p}=0.043, \mathrm{~B}=0.087, \mathrm{p}=0.047$, respectively).

\section{DISCUSSION}

In this study, we aimed to investigate the effects of self-conscious- ness on insomnia symptoms. Social anxiety was higher in poor sleepers than in good sleepers, while other self-consciousness domains showed no significant difference between the two groups. Among the three self-consciousness domains, only social anxiety showed significant correlation with poor sleepers. In multiple regression analysis for each insomnia scale, social anxiety was significantly associated with poor sleepers with both high PSQI and ISI score, while private self-consciousness was negatively correlated with poor sleepers with high ISI score.

The most notable result of our study is that social anxiety among the three self-consciousness domains was found to have significant correlation with insomnia symptoms. The association of insomnia symptoms with social anxiety could be explained by that emotional distress and hyperarousal caused by high self-con-

Table 2. Association of factors including self-consciousness with poor sleepers ${ }^{a}$

\begin{tabular}{|c|c|c|c|}
\hline Variables & B & $\mathrm{p}$ & OR $(95 \% \mathrm{CI})$ \\
\hline Age (yr) & -0.081 & 0.156 & $0.922(0.824-1.031)$ \\
\hline Sex, female & 1.172 & 0.183 & $3.227(0.575-18.096)$ \\
\hline Weight (kg) & 0.052 & 0.123 & $1.504(0.986-1.126)$ \\
\hline Education (yr) & 0.171 & 0.344 & $1.186(0.833-1.689)$ \\
\hline \multicolumn{4}{|l|}{ Current job status } \\
\hline Regular worker & Reference & & \\
\hline Part-time job & 20.613 & 1.000 & $895768629.60\left(0.000^{-}\right)$ \\
\hline Housemaker & -19.286 & 1.000 & $0.000\left(0.000^{-}\right)$ \\
\hline Student & -0.961 & 0.277 & $0.383(0.068-2.165)$ \\
\hline Inoccupation & 19.883 & 1.000 & $431398599.63\left(0.000^{-}\right)$ \\
\hline Total nap time (min) & -0.008 & 0.566 & $0.992(0.964-1.021)$ \\
\hline Amount of drinks (g) & 0.052 & 0.399 & $1.054(0.933-1.190)$ \\
\hline Private self-consciousness ${ }^{\mathrm{b}}$ & -0.131 & 0.107 & $0.877(0.748-1.029)$ \\
\hline Public self-consciousness ${ }^{\mathrm{b}}$ & 0.028 & 0.660 & $1.029(0.907-1.167)$ \\
\hline Social anxiety ${ }^{\mathrm{b}}$ & 0.087 & $0.046^{*}$ & $1.091(1.001-1.189)$ \\
\hline
\end{tabular}

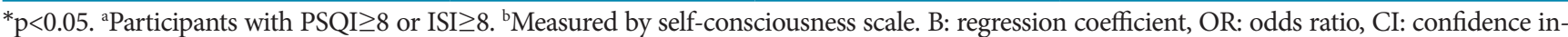
terval, PSQI: Pittsburgh Sleep Quality Index, ISI: Insomnia Severity Index

Table 3. Association of factors including self-consciousness with insomnia scales

\begin{tabular}{|c|c|c|c|c|c|c|}
\hline & \multicolumn{3}{|c|}{$\mathrm{PSQI}^{\mathrm{a}}$} & \multicolumn{3}{|c|}{$\mathrm{ISI}^{\mathrm{b}}$} \\
\hline & B & SE & $\mathrm{p}$ & B & SE & $\mathrm{p}$ \\
\hline Age (yr) & -0.083 & 0.048 & 0.086 & -0.107 & 0.073 & 0.148 \\
\hline Sex, female & 1.658 & 0.756 & $0.032^{*}$ & 1.708 & 1.157 & 0.145 \\
\hline Weight (kg) & 0.055 & 0.028 & 0.053 & 0.021 & 0.043 & 0.624 \\
\hline Education (yr) & 0.165 & 0.165 & 0.321 & 0.136 & 0.253 & 0.594 \\
\hline \multicolumn{7}{|l|}{ Current job status } \\
\hline Regular worker & Reference & & & Reference & & \\
\hline Part-time job & 1.618 & 2.266 & 0.478 & 0.188 & 3.258 & 0.954 \\
\hline Housemaker & 1.604 & 2.492 & 0.522 & -0.211 & 3.492 & 0.942 \\
\hline Student & -0.421 & 0.831 & 0.614 & -0.092 & 1.199 & 0.958 \\
\hline Inoccupation & 0.540 & 2.316 & 0.816 & 4.594 & 3.330 & 0.173 \\
\hline Total nap time (min) & 0.006 & 0.013 & 0.657 & 0.003 & 0.020 & 0.893 \\
\hline Amount of drinks (g) & 0.090 & 0.053 & 0.094 & 0.104 & 0.077 & 0.181 \\
\hline Private self-consciousness ${ }^{c}$ & -0.098 & 0.064 & 0.130 & -0.202 & 0.098 & $0.043^{*}$ \\
\hline Public self-consciousness ${ }^{c}$ & -0.046 & 0.058 & 0.424 & -0.032 & 0.088 & 0.723 \\
\hline Social anxiety ${ }^{c}$ & 0.091 & 0.028 & $0.002^{\dagger}$ & 0.087 & 0.043 & $0.047^{*}$ \\
\hline
\end{tabular}

${ }^{*} \mathrm{p}<0.05,{ }^{\dagger} \mathrm{p}<0.01$. ${ }^{\mathrm{P} S Q} \mathrm{I} \geq 8$. ${ }^{\mathrm{b} I S I} \geq 8$. ${ }^{\mathrm{c}}$ Measured by self-consciousness scale. B: regression coefficient, SE: standard error, PSQI: Pittsburgh Sleep Quality Index, ISI: Insomnia Severity Index 
sciousness. Emotional distress, perpetuating worry, and physiological hyperarousal could produce insomnia symptoms [28,29]. Previous literatures reported that high level of self-consciousness contribute to hyperarousal $[30,31]$. However, we should note that self-consciousness is not always associated with psychopathological status, as highly self-conscious individuals are aware of their attitudes which could lead to self-regulation and psychological wellbeing $[32,33]$. The reason of only social anxiety showed significant correlation to poor sleepers might because social anxiety is the domain with the highest correlation to emotional distress and psychiatric symptoms including depression, anxiety, and interpersonal sensitivity among three self-consciousness domains [34]. We can assume that high self-consciousness with emotional distress could be related to worry or hyperarousal which could affect insomnia symptoms.

Private self-consciousness showed negative association with poor sleepers only with high ISI. We could assume this is because private self-consciousness is more likely to be associated with beneficial mental effect than other domains of self-consciousness. A previous study reported that private self-consciousness did not have association with various measures of anxiety, while public self-consciousness did [35]. There are also studies about beneficial mental effect of private self-consciousness including tolerance to stress, relaxation, and daily well-being [36,37]. Yet, it is difficult to confirm if there is a beneficial effect of private self-consciousness to insomnia symptoms solely based on the result of this study. In this study, private self-consciousness showed a significant correlation only with ISI score of 8 or high, not with PSQI score or poor sleepers. Further study about relationship between self-consciousness and insomnia is needed to verify this.

What should be noted here is that no significant difference in the objective sleep parameters was found between the poor sleepers and the good sleepers. This is because we recruited healthy population for this study. Poor sleepers in this study was defined as high PSQI or ISI score among healthy population. The most significantly different sleep parameter between poor sleepers and good sleepers was subjective sleep quality, which could explain high PSQI or ISI score of participants even without disturbance of other sleep parameters.

There are several limitations of this study. First, the survey on self-consciousness was performed after the end of experiment. Since sleep parameters and SCS are not measured at the same time, it might be difficult to confirm the effects of self-consciousness on insomnia symptoms. However, self-consciousness is an adaptive personality processes of individuals that is expected to be not easily changed. Previous study on self-consciousness reported SCS showed year-to-year stability [38]. Thus, we could assume that self-consciousness of the participants constantly remained during the experiment periods. Second, number of sample size was small. It is possible that small number of sample size could led to the bias of sample characteristics.

This is the first study to evaluate the effects of self-consciousness on insomnia symptoms. We found the correlation between high anxiety and poor sleep. The result of this study indicates that high self-consciousness with emotional distress could affect insomnia symptoms.

\section{Acknowledgments}

This study was funded by the Basic Science Research Program through the National Research Foundation of Korea (NRF); the Ministry of Science, ICT \& Future Planning, Republic of Korea (grant number: 2017R1A2B3008214 to Eun Lee); and the Intelligence Information Expansion Support System for Private Organizations supervised by the National IT Industry Promotion Agency (grant number: A0602-19-1020 to Eun Lee).

\section{Conflicts of Interest}

The authors have no potential conflicts of interest to disclose.

\section{Author Contributions}

Conceptualization: all authors. Data curation: all authors. Formal analysis: Min Su Kim, Eun Lee, Kyung Mee Park, Investigation: Min Su Kim, Eun Lee, Kyung Mee Park. Methodology: Min Su Kim, Eun Lee, Kyung Mee Park. Project administration: all authors. Resources: all authors. Software: Min Su Kim, Hye Yoon Park, Eunchong Seo, Eun Lee. Supervision: Eun Lee, Suk Kyoon An, Kyung Mee Park. Validation: Min Su Kim, Eun Lee, Kyung Mee Park. Visualization: Min Su Kim, Eun Lee, Kyung Mee Park. Writing-original draft: Min Su Kim, Kyung Mee Park. Writing—review \& editing: all authors.

\section{ORCID iDs}

\section{Min Su Kim (1)}

https://orcid.org/0000-0003-0940-7504

Eun Lee (1)

https://orcid.org/0000-0002-7462-0144

Hye Yoon Park (1)

https://orcid.org/0000-0001-9579-8112

Eunchong Seo (1)

https://orcid.org/0000-0003-2644-266X

Suk Kyoon An (10)

https://orcid.org/0000-0003-4576-6184

Kyung Mee Park (1)

https://orcid.org/0000-0002-2416-2683

\section{REFERENCES}

1. Gallup K. Korean Gallup survey on sleep and dream. 2017. Available at: https://www.gallup.co.kr/gallupdb/reportContent.asp?seqNo=846. Accessed February 13, 2021.

2. Stranges S, Tigbe W, Gómez-Olivé FX, Thorogood M, Kandala NB. Sleep problems: an emerging global epidemic? Findings from the INDEPTH WHO-SAGE study among more than 40,000 older adults from 8 countries across Africa and Asia. Sleep 2012;35:1173-1181.

3. Sofi F, Cesari F, Casini A, Macchi C, Abbate R, Gensini GF. Insomnia and risk of cardiovascular disease: a meta-analysis. Eur J Prev Cardiol 2014;21: 57-64.

4. Heslop P, Smith GD, Metcalfe C, Macleod J, Hart C. Sleep duration and mortality: the effect of short or long sleep duration on cardiovascular and all- 
cause mortality in working men and women. Sleep Med 2002;3:305-314.

5. Zhai L, Zhang H, Zhang D. Sleep duration and depression among adults: a meta-analysis of prospective studies. Depress Anxiety 2015;32:664-670.

6. Riemann D, Voderholzer U. Primary insomnia: a risk factor to develop depression? J Affect Disord 2003;76:255-259.

7. Nebes RD, Buysse DJ, Halligan EM, Houck PR, Monk TH. Self-reported sleep quality predicts poor cognitive performance in healthy older adults. J Gerontol B Psychol Sci Soc Sci 2009;64:180-187.

8. Martin SE, Engleman HM, Deary IJ, Douglas NJ. The effect of sleep fragmentation on daytime function. Am J Respir Crit Care Med 1996;153(4 Pt 1):1328-1332.

9. Duval S, Wicklund RA. A theory of objective self awareness. Cambridge, MA: Academic Press; 1972.

10. Benjamins JS, Migliorati F, Dekker K, Wassing R, Moens S, Blanken TF, et al. Insomnia heterogeneity: characteristics to consider for data-driven multivariate subtyping. Sleep Med Rev 2017;36:71-81.

11. Blankstein KR, Flett GL, Watson MS, Koledin S. Test anxiety, self-evaluative worry, and sleep disturbance in college students. Anxiety Res 1990;3:193-204.

12. Fenigstein A, Scheier MF, Buss AH. Public and private self-consciousness: assessment and theory. J Consult Clin Psychol 1975;43:522-527.

13. DaSilveira A, DeSouza ML, Gomes WB. Self-consciousness concept and assessment in self-report measures. Front Psychol 2015;6:930.

14. Buckner JD, Bernert RA, Cromer KR, Joiner TE, Schmidt NB. Social anxiety and insomnia: the mediating role of depressive symptoms. Depress Anxiety $2008 ; 25: 124-130$.

15. Schmidt RE, Van der Linden M. The aftermath of rash action: sleep-interfering counterfactual thoughts and emotions. Emotion 2009;9:549-553.

16. Raffray T, Bond TL, Pelissolo A. Correlates of insomnia in patients with social phobia: role of depression and anxiety. Psychiatry Res 2011;189:315-317.

17. First MB, Williams JBW, Karg RS, Spitzer RL. Structured clinical interview for DSM-5-research version (SCID-5 for DSM-5, research version; SCID5-RV). Arlington, VA: American Psychiatric Association; 2015.

18. Buysse DJ, Reynolds CF 3rd, Monk TH, Berman SR, Kupfer DJ. The Pittsburgh Sleep Quality Index: a new instrument for psychiatric practice and research. Psychiatry Res 1989;28:193-213.

19. Grandner MA, Kripke DF, Yoon IY, Youngstedt SD. Criterion validity of the Pittsburgh Sleep Quality Index: investigation in a non-clinical sample. Sleep Biol Rhythms 2006;4:129-139.

20. Carpenter JS, Andrykowski MA. Psychometric evaluation of the Pittsburgh Sleep Quality Index. J Psychosom Res 1998;45:5-13.

21. Fictenberg NL, Putnam SH, Mann NR, Zafonte RD, Millard AE. Insomnia screening in postacute traumatic brain injury: utility and validity of the Pitts- burgh Sleep Quality Index. Am J Phys Med Rehabil 2001;80:339-345.

22. Bastien $\mathrm{CH}$, Vallières A, Morin CM. Validation of the Insomnia Severity Index as an outcome measure for insomnia research. Sleep Med 2001;2:297-307.

23. Morin CM, Belleville G, Bélanger L, Ivers H. The Insomnia Severity Index: psychometric indicators to detect insomnia cases and evaluate treatment response. Sleep 2011;34:601-608.

24. Cole RJ, Kripke DF, Gruen W, Mullaney DJ, Gillin JC. Automatic sleep/wake identification from wrist activity. Sleep 1992;15:461-469.

25. Carney CE, Buysse DJ, Ancoli-Israel S, Edinger JD, Krystal AD, Lichstein $\mathrm{KL}$, et al. The consensus sleep diary: standardizing prospective sleep selfmonitoring. Sleep 2012;35:287-302.

26. Fenigstein A. Self-consciousness, self-attention, and social interaction. J Pers Soc Psychol1979;37:75-86.

27. Nie NH, Bent DH, Hull CH. SPSS: statistical package for the social sciences. New York: McGraw-Hill; 1975.

28. Bonnet MH, Arand DL. Hyperarousal and insomnia: state of the science. Sleep Med Rev 2010;14:9-15.

29. Spielman AJ, Caruso LS, Glovinsky PB. A behavioral perspective on insomnia treatment. Psychiatr Clin North Am 1987;10:541-553.

30. Wassing R, Benjamins JS, Dekker K, Moens S, Spiegelhalder K, Feige B, et al. Slow dissolving of emotional distress contributes to hyperarousal. Proc Natl Acad Sci U S A 2016;113:2538-2543.

31. Wells A. A multi-dimensional measure of worry: development and preliminary validation of the anxious thoughts inventory. Anxiety Stress Coping 1994;6:289-299.

32. Scheier MF. Effects of public and private self-consciousness on the public expression of personal beliefs. J Pers Soc Psychol 1980;39:514-521.

33. Lischetzke T, Eid M. Is attention to feelings beneficial or detrimental to affective well-being? Mood regulation as a moderator variable. Emotion 2003; 3:361-377.

34. Panayiotoua G, Kokkinosb CM. Self-consciousness and psychological distress: a study using the Greek SCS. Pers Individ Differ 2006;41:83-93.

35. Saboonchi F, Lundh LG. Perfectionism, self-consciousness and anxiety. Pers Individ Differ 1997;22:921-928.

36. Mullen B, Suls J. "Know thyself": Stressful life changes and the ameliorative effect of private self-consciousness. J Exp Soc Psychol 1982;18:43-55.

37. Newman DB, Nezlek JB. Private self-consciousness in daily life: Relationships between rumination and reflection and well-being, and meaning in daily life. Pers Individ Differ 2019;136:184-189.

38. Davis MH, Franzoi SL. Stability and change in adolescent self-consciousness and empathy. J Res Pers 1991;25:70-87. 